\title{
Survival Analysis of Patients with Inflammatory Breast Cancer in Relation to Clinical and Histopathological Characteristics
}

This article was published in the following Dove Press journal: Cancer Management and Research

\author{
Marius Preda ${ }^{1,2}$ \\ Răzvan Ilina ${ }^{1,2}$ \\ Ovidiu Potre 3,4 \\ Cristina Potre 3,4 \\ Octavian Mazilu' ${ }^{1,2}$ \\ 'Ist Department of Surgery, Discipline of \\ Surgical Semiology II, Victor Babes \\ University of Medicine and Pharmacy, \\ Timisoara, Romania; ${ }^{2}$ 2nd General and \\ Oncological Surgery Clinic, Timisoara's \\ Emergency City Hospital, Timisoara, \\ Romania; ${ }^{3}$ Department of Internal \\ Medicine, Victor Babes University of \\ Medicine and Pharmacy, Timisoara, \\ Romania; ${ }^{4}$ Hematology Clinic, \\ Timisoara's Emergency City Hospital, \\ Timisoara, Romania
}

Purpose: To evaluate the survival of patients with inflammatory breast cancer (IBC) and to correlate these survival rates with the histopathological parameters found in the resection specimen of the tumor.

Patients and Methods: This retrospective study was based on 27 patients that had been diagnosed and had undergone surgery in the 2nd General and Oncological Surgery Clinic of the County Emergency Hospital in Timisoara, Romania. Data about the patient group were collected from archived patient files over a period of eight years starting from January 2008. The collected data regarded age, tumor size and histopathological type and immunohistochemistry (IHC), presence or absence of tumor embolus in lymphatic or blood vessels and the presence or absence of distant metastases at the time of diagnosis. We evaluated the impact of tumor characteristics on the patients' outcome.

Results: The 12-month survival rates postsurgery were significantly increased if tumors were $<5 \mathrm{~cm}$ compared to those $>5 \mathrm{~cm}(p=0.046)$, if nodal status was N0 vs N1-2 ( $p=0.039)$, as well as in cases where distant metastases were absent, compared to patients with distant metastases $(p=0.001, \alpha=0.001)$ and positive-hormone receptors $(p=0.043)$. Survival was influenced neither by histopathological type $(p=0.357)$ nor by the presence of tumor embolus in the resection specimen $(p=0.250)$ and HER2 status $(p=0.763)$. Survival at 12 months after surgery was equal between those with stage IIIB noninflammatory breast cancer (NIBC) and IBC. At 24 months after surgery, IBC cases presented a significantly lower probability of survival.

Conclusion: The prognosis of patients with IBC is reserved compared to NIBC, increased tumor size, positive lymph node, hormone receptors negative cases and distant metastases drastically decreasing survival rates.

Keywords: inflammatory breast cancer, diagnosis, survival, prognosis

\section{Introduction}

Inflammatory breast cancer (IBC) or carcinomatous mastitis is the most aggressive form of breast cancer. It is a rare type of cancer with an incidence of 1 to $5 \%$ that determines different clinical manifestations compared to other types of breast cancer, thus proving more difficult to diagnose. ${ }^{1}$

It is characterized by erythema, skin ridging with characteristic peau d'orange secondary to dermal lymphatic tumor involvement, rapid enlargement of the breast. Clinical diagnosis combines observations of the affected breast, medical history and physical examination. Because of its behavior, IBC can be easily misdiagnosed or
Correspondence: Răzvan Ilina No. 5 Gheorghe Dima Street, 2nd General and Oncological Surgery Clinic Timisoara's Emergency City Hospital,

Timisoara, Romania

Tel/Fax +40 749257424

Email razvanilina@yahoo.co.uk 
treated inappropriately. There may be nipple discharge, or the nipple may appear inverted or flattened. Tumor may or may not be present. These nonspecific characteristics can ultimately result in delayed diagnosis, and other breast diseases can be confused with IBC.

The presence of GCC haplotype may increase the risk of disease development and might contribute, besides other factors, in the overexpression of IL 10 gene in aggressive breast cancer phenotype IBC. ${ }^{2}$

Despite major efforts to understand IBC biology, genomic studies have not led to the discovery of a biological mechanism in IBC that can be translated into novel therapeutic strategies. ${ }^{3}$ Several studies show the importance of using preclinical models to better understand tumor biology, with good results. ${ }^{4}$

Carcinomatous mastitis is significantly more aggressive, due to its accelerated growth and dissemination to other organs, more so than other types of breast cancer. Consequently, women diagnosed with this form of cancer have a critical prognosis. Moreover, the IBC stage at diagnosis is at least IIIB, sometimes even IV, this factor carrying a negative prognosis on its own.

Compared to other breast cancer forms, late diagnosis, as well as a proclivity towards accelerated growth and metastasis, render its treatment difficult and seldom successful.

The objective of this study was to evaluate the results of 12-month and 24-month survival probability of patients with IBC after diagnosis, as well as to correlate the survival rate of IBC patients with tumor size, histological type of cancer, lymph node status, the presence or absence of metastasis at the time of diagnosis, the presence of tumor emboli, hormone receptor and HER2 status. Survival rates of patients with IBC and of those with noninflammatory breast cancer NIBC) of similar stage were also analyzed.

\section{Patients and Methods}

\section{Study Design and Patients}

This retrospective study includes 27 patients with carcinomatous mastitis from a total of 1193 patients diagnosed with breast cancer in the 2nd General and Oncological Surgery Department of the Timisoara County Emergency Clinical Hospital, Romania, for a period of eight years, from January 1, 2008 until December 31, 2015.

The study was approved by the Ethics Commission of the Timisoara Municipal Hospital, and all enrolled patients signed the agreement of participation in the study.
Detailed analysis of archived patient files led to the selection of these patients, along with surgical protocols and histopathological reports. Inclusion criteria contemplated in this study were the clinical diagnosis of carcinomatous mastitis supported by the histopathological exam and IHC performed on the biopsy specimen. If the HER2 result was equivocal, fluorescence in situ hybridization or chromogenic in situ hybridization was performed. The staging of the disease was established by applying the TNM criteria and histologic grading using SBR grading system.

After the histopathological confirmation, all patients were directed to the Oncology Clinic for neoadjuvant chemotherapy treatment. Twenty patients followed four courses with epirubicin $75 \mathrm{mg} / \mathrm{m}^{2}$ and cyclophosphamide $600 \mathrm{mg} / \mathrm{m}^{2}$ every three weeks, followed by four courses with paclitaxel $175 \mathrm{mg} / \mathrm{m}^{2}$. In seven cases, patients over 65 years with comorbidities, the neoadjuvant chemotherapy was docetaxel $75 \mathrm{mg} / \mathrm{m}^{2}$ and carboplatin AUC 5-6 for four courses, every three weeks.

Either a Madden or a modified Patey radical mastectomy was performed 21-30 days following chemotherapy. In order to obtain local disease control, a simple mastectomy was performed in patients with stage IV cancer.

All patients received adjuvant radiotherapy treatment.

Fifteen patients with positive hormone receptors were given adjuvant treatment with tamoxifen for two years, followed by aromatase inhibitors for three years.

In case of HER2-positive, adjuvant treatment with trastuzumab was administered for one year, induction dose of $8 \mathrm{mg} / \mathrm{kg}$, then $6 \mathrm{mg} / \mathrm{kg}$ every three weeks. In our country, trastuzumab has only been administered as a neoadjuvant treatment combined with chemotherapy in HER2-positive cases since 2016.

The 24-month follow-up was made available to all patients. Survival statistics were observed in relation to tumor size, histopathological type, lymph node status, the presence or absence of distant metastases at diagnosis and the presence or absence of tumor embolus, presence or absence of hormone receptors, HER2+ status. A comparison of survival probability between patients with stage III IBC and NIBC was performed.

The data describing the histopathological type of tumor and ypTNM classification was acquired from histopathological reports.

A statistical analysis of the data was conducted 24 months after the last patient was included in the study. 


\section{Statistics}

Data was collected and analyzed using Epi Info v. 7.

Survival analysis was performed in relation to time through a graphical presentation of cumulative survival rate $\mathrm{Pi}=\mathrm{f}(\mathrm{i})$ or cumulative mortality rate $\mathrm{Qi}=1-\mathrm{Pi}=\mathrm{g}$ (i) and Kaplan-Meier curves. For comparing two survival rates, log rank test was used.

\section{Results}

The total number of breast cancer cases in the study period was 1193, 27 of which were IBC, accounting for $2.26 \%$.

The age of patients with IBC was between 34 and 75 years with a mean age of 57.5 years. The mean age of patients with NIBC was 66 years.

After performing the histopathological examination of the biopsies, out of the total number of cases, 20 (74.08\%) were diagnosed with infiltrating ductal carcinoma (Figure $1 \mathrm{~A}$ and $\mathrm{B})$, one $(3.70 \%)$ patient with infiltrating ductal and lobular carcinoma (admixed type), and six (22.22\%) with lobular carcinoma.

After neoadjuvant chemotherapy, the biopsy specimens (Table 1) showed one case (3.7\%) T1, 19 (70.37\%) cases $\mathrm{T} 2$, four $(14.81 \%)$ cases T3 and three $(11.11 \%)$ cases T4; four cases (14.8\%) pN0 and 23 cases pN1-N2 (85.2\%); 15 $(55.55 \%)$ hormone receptor-positive cases and 12 hormone receptor-negative cases (44.44\%); eight (29.62\%) HER2+ positive, 19 (70.37\%) HER2+ negative cases. Furthermore, five patients $(18.51 \%)$ had triple negative cancer form. Tumor emboli were identified in 10 excised tumors $(37.04 \%)$ after neoadjuvant chemotherapy. Distant metastases M1 were identified in two cases $(7.40 \%)$, with pulmonary localization.

By July 2018, 14 (51.85\%) out of the 27 patients with IBC were deceased. Statistical analysis showed that the average survival period postsurgery was 14 months.

In seven of the cases, the patients $(26 \%)$ presented with a tumor $>5 \mathrm{~cm}$, and 20 cases presented with tumors $<5 \mathrm{~cm}(74 \%)$.

The survival probability was significantly increased in cases where the size of the tumor was $<5 \mathrm{~cm}$ (log rank Mantel-Cox test, $p=0.046, \alpha=0.05$ ), namely $68 \%$ compared to $48 \%$ for tumors $>5 \mathrm{~cm}$. The relation between survivability and tumor dimension can be observed in Figure 2.

The survival probability 12 months after surgery was $28 \%$ for patients with infiltrating ductal carcinoma and $42 \%$ for infiltrating lobular carcinoma, data summarized in Figure 3. There were no statistically significant
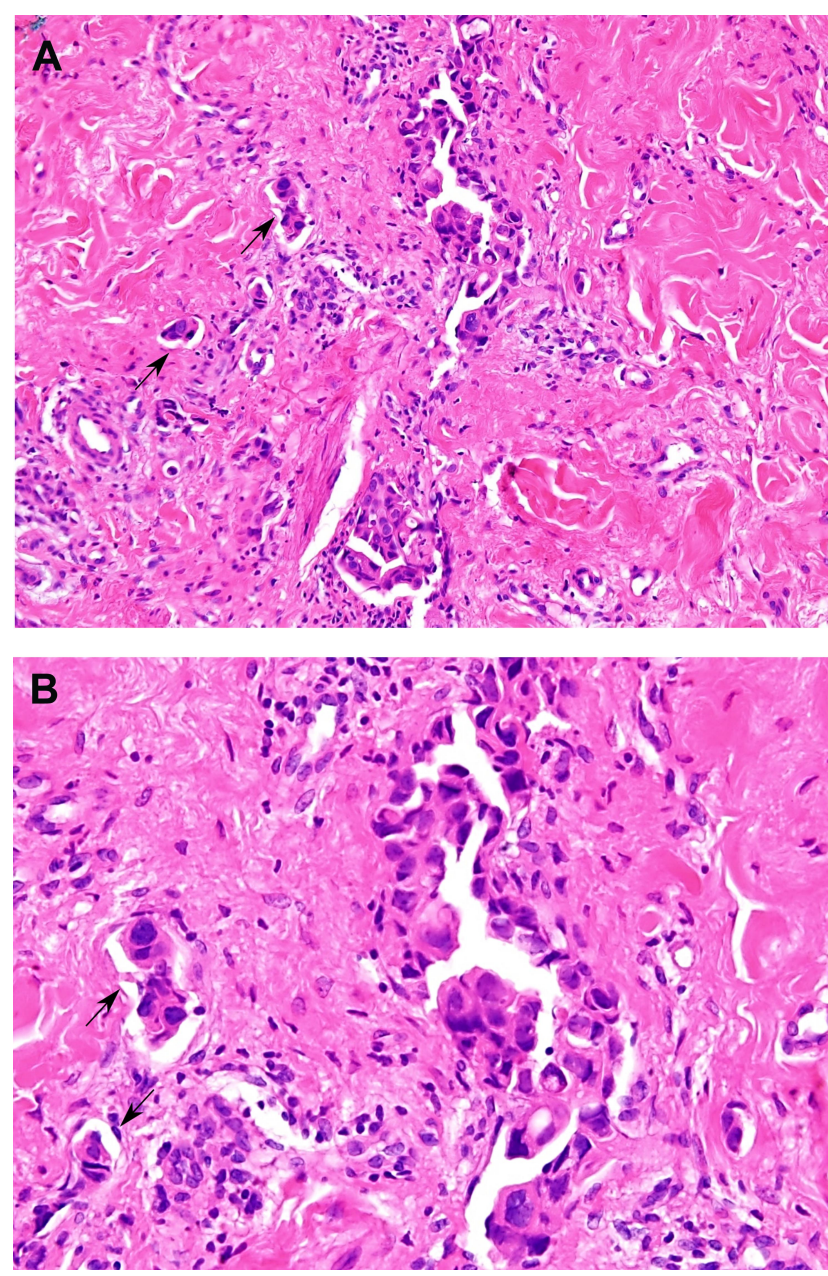

Figure I (A and B) Invasive ductal breast carcinoma (NST) clinically diagnosed as inflammatory breast carcinoma. Tumor cells invading dermal lymphatic vessels (arrows). (A) - H\&Ex20 and (B) - H\&Ex40 (pictures from Clinic Archive).

Abbreviations: IBC, inflammatory breast cancer; NIBC, noninflammatory breast cancer; NST, nonspecial type.

differences between the two aforementioned groups regarding their survival curves (log rank Mantel-Cox test, $p=0.299, \alpha=0.05$ ).

A significantly higher survival probability was identified in cases with lymph node status N0 (log rank MantelCox test, $p=0.039, \alpha=0.05$ ), where the 12-month survival probability was $32 \%$ for patients with N1-N2 lymph node status, and $70 \%$ for cases with N0 lymph node status (Figure 4). The survival probability at 20 months was $40 \%$ in patients with N0 lymph node status, while that of those with N1-N2 could not be calculated due to their short survival period.

The 12-month survival probability was $61 \%$ for patients with M0, while at 20 months it was 34\% (Figure $5)$. In two cases (7.4\%) with extensive metastasizing the calculation of the 12-month survival probability could not 
Table I Histopathological and IHC Parameters of Cases

\begin{tabular}{|c|c|c|c|c|c|c|c|c|c|c|}
\hline & Age & HP Type & $\mathbf{T}$ & $\mathbf{N}$ & $M$ & Tumor Emboli & ER & PR & K167 & HER2 Status \\
\hline I. & 58 & IDC SBR II & $\mathrm{TI}$ & N2 & MO & LIVI & $0 \%$ & $0 \%$ & $40 \%$ & Negative \\
\hline 2. & 65 & IDC SBR II & $\mathrm{T} 2$ & No & Mo & LOVO & $80 \%$ & $60 \%$ & $10 \%$ & Negative \\
\hline 3. & 63 & IDC SBR III & $\mathrm{T} 2$ & N2 & MO & LIVI & $98 \%$ & $40 \%$ & $20 \%$ & Negative \\
\hline 4. & 74 & IDC SBR II & $\mathrm{T} 2$ & NI & Mo & LIVI & $70 \%$ & $0 \%$ & $20 \%$ & Negative \\
\hline 5. & 55 & IDC SBR II & $\mathrm{T} 2$ & No & MO & LIVI & $90 \%$ & $10 \%$ & $60 \%$ & Negative \\
\hline 6. & 75 & IDC SBR II & $\mathrm{T} 2$ & N2 & MI P & LOVO & $80 \%$ & $0 \%$ & $10 \%$ & Negative \\
\hline 7. & 51 & IDC SBR II & $\mathrm{T} 2$ & N2 & Mo & LOVO & $70 \%$ & $0 \%$ & $14 \%$ & Negative \\
\hline 8. & 56 & IDC SBR II & $\mathrm{T} 2$ & N2 & MO & LOVO & $0 \%$ & $0 \%$ & $20 \%$ & Negative \\
\hline 9. & 67 & IDC SBR II & $\mathrm{T} 2$ & No & Mo & LOVO & $80 \%$ & $75 \%$ & $15 \%$ & Negative \\
\hline 10. & 55 & IDC SBR II & $\mathrm{T} 2$ & N2 & MO & LOVO & $90 \%$ & $0 \%$ & $30 \%$ & Positive \\
\hline 11. & 50 & IDC SBR III & $\mathrm{T} 3$ & N2 & Mo & LIVI & $0 \%$ & $0 \%$ & $60 \%$ & Negative \\
\hline 12. & 42 & IDC SBR II & $\mathrm{T} 2$ & N2 & MO & LOVO & $60 \%$ & $0 \%$ & $40 \%$ & Negative \\
\hline 13. & 71 & IDC SBR II & $\mathrm{T} 2$ & N2 & Mo & LOVO & $98 \%$ & $50 \%$ & $40 \%$ & Positive \\
\hline 14. & 65 & IDC SBR II & $\mathrm{T} 2$ & N2 & Mo & LIVI & $0 \%$ & $0 \%$ & $90 \%$ & Positive \\
\hline 15. & 52 & IDC SBR II & T3 & NI & Mo & LOVO & $0 \%$ & $0 \%$ & $60 \%$ & Positive \\
\hline 16. & 44 & ILC SBR II & T3 & NI & MI P & LOVO & $0 \%$ & $0 \%$ & $50 \%$ & Negative \\
\hline 17. & 59 & ILC SBR II & $\mathrm{T} 4$ & N2 & MO & LOVO & $70 \%$ & $40 \%$ & $30 \%$ & Negative \\
\hline 18. & 48 & IDC SBR II & $\mathrm{T} 2$ & No & MO & LOVO & $80 \%$ & $70 \%$ & $30 \%$ & Negative \\
\hline 19. & 49 & IDC SBR II & $\mathrm{T} 3$ & N2 & Mo & LOVO & $0 \%$ & $0 \%$ & $60 \%$ & Positive \\
\hline 20. & 53 & ILC SBR II & $\mathrm{T} 3$ & N2 & Mo & LOVO & $0 \%$ & $0 \%$ & $30 \%$ & Positive \\
\hline 21. & 47 & IDC SBR II & $\mathrm{T} 2$ & N2 & Mo & LOVO & $0 \%$ & $0 \%$ & $10 \%$ & Negative \\
\hline 22. & 67 & ILC SBR II & $\mathrm{T} 2$ & N2 & MO & LOVO & $0 \%$ & $0 \%$ & $40 \%$ & Positive \\
\hline 23. & 68 & IDC SBR II & $\mathrm{T} 2$ & N2 & Mo & LIVI & $10 \%$ & $40 \%$ & $1 \%$ & Negative \\
\hline 24. & 34 & Admixed cc SBR II & $\mathrm{T} 2$ & N2 & Mo & LIVI & $60 \%$ & $20 \%$ & $30 \%$ & Negative \\
\hline 25. & 61 & IDC SBR II & $\mathrm{T} 2$ & $\mathrm{NI}$ & Mo & LOVO & $0 \%$ & $0 \%$ & $10 \%$ & Positive \\
\hline 26. & 75 & ILC SBR II & $\mathrm{T} 3$ & N2 & Mo & LIVI & $0 \%$ & $0 \%$ & $30 \%$ & Negative \\
\hline 27. & 51 & ILC SBR II & $\mathrm{T} 2$ & N2 & Mo & LIVI & $70 \%$ & $40 \%$ & $10 \%$ & Negative \\
\hline
\end{tabular}

be performed, as a consequence of their death at two and three months, respectively postmastectomy. Statistically significant differences between the two survival curves can be observed (Mantel-Cox log rank test, $p<0.001$, $\alpha=0.001$ ).

There were no statistically significant differences between the two survival curves dependent on the presence/absence of tumor emboli (Mantel-Cox log rank test, $p=0.25, \alpha=0.05$ ), the survival probability at 12 months being $60 \%$ for patients who presented with tumor emboli and $62 \%$ for those who did not (Figure 6).

There were significant differences between the two survival curves dependent on the presence or absence of hormone receptors, $\log$ rank (Mantel-Cox), $p=0.043$. Twenty months after surgery, the survival probability in cases with negative receptors was $18 \%$, while, in patients with positive receptors the survival probability was $36 \%$ (Figure 7 ).

There were no statistically significant differences between the two survival curves dependent on the overexpression of the HER2 (Figure 8). The differences between the two Kaplan-Meier curves are insignificantlog rank (Mantel-Cox), $p=0.763$.

In order to further investigate survivability in patients with IBC, a comparison between those patients and 27 patients with stage IIIB NIBC was studied.

The survival probability at 12 months was $50 \%$ for the group with N0 IBC and $68 \%$ for the group with stage IIIB NIBC, with no statistically significant differences (MantelCox $\log$ rank test, $p=0.948, \alpha=0.05$ ). At 24 months, the survival probability was $8 \%$ for patients with IBC and $20 \%$ for those with stage IIIB NIBC, this difference, however, being of statistical significance (Mantel-Cox log rank test, $p=0.046)$. These data are represented graphically in Figure 9.

\section{Discussion}

Carcinomatous mastitis is a rare, aggressive, and fatal form of IBC, which requires a multimodal approach. ${ }^{5}$ All patients received treatment according to the therapeutic guidelines. The treatment sequence was neoadjuvant chemotherapy, surgery, adjuvant radiotherapy, adjuvant chemotherapy, 


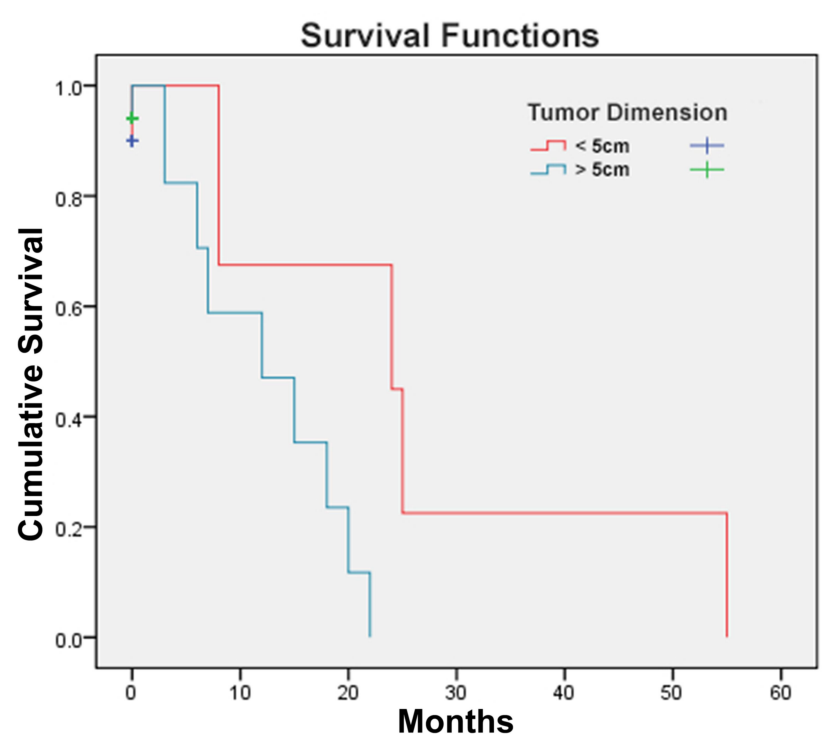

Figure 2 The survival probability according to tumor dimension.

\section{Survival Functions}

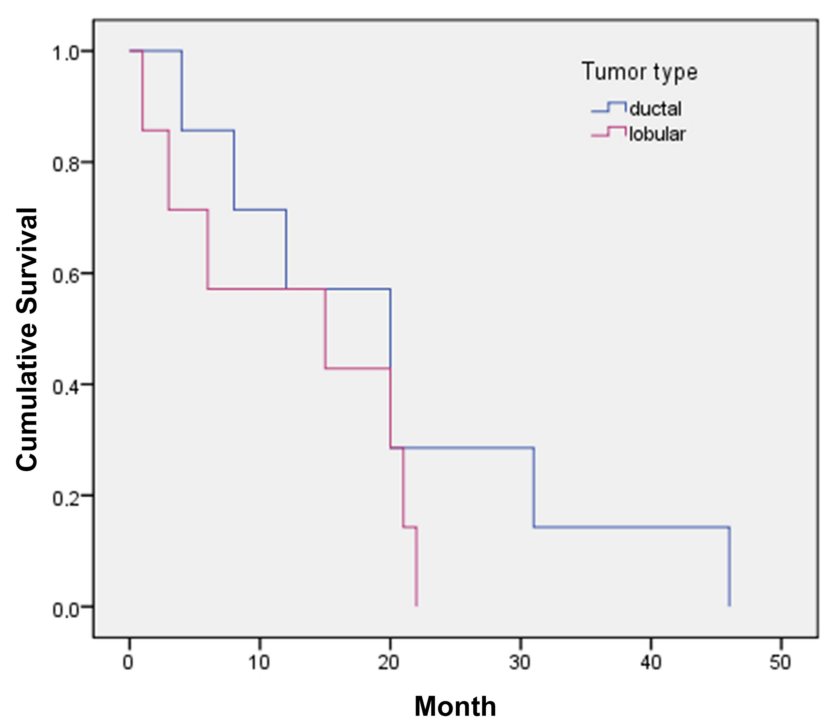

Figure 3 The survival probability according to histopathological type.

followed by targeted antihormonal treatment in hormonedependent cases and trastuzumab in HER2+ positive ones.

The surgical procedures were Madden and Patey mastectomies. The modified radical mastectomy is also recommended by other authors. ${ }^{6}$ There are a number of authors claiming that in IBC treated with neoadjuvant chemotherapy that responds favorably, conservative interventions can be performed in selected cases. ${ }^{7}$

There are several inflammatory breast diseases that present the same clinical manifestation leading to

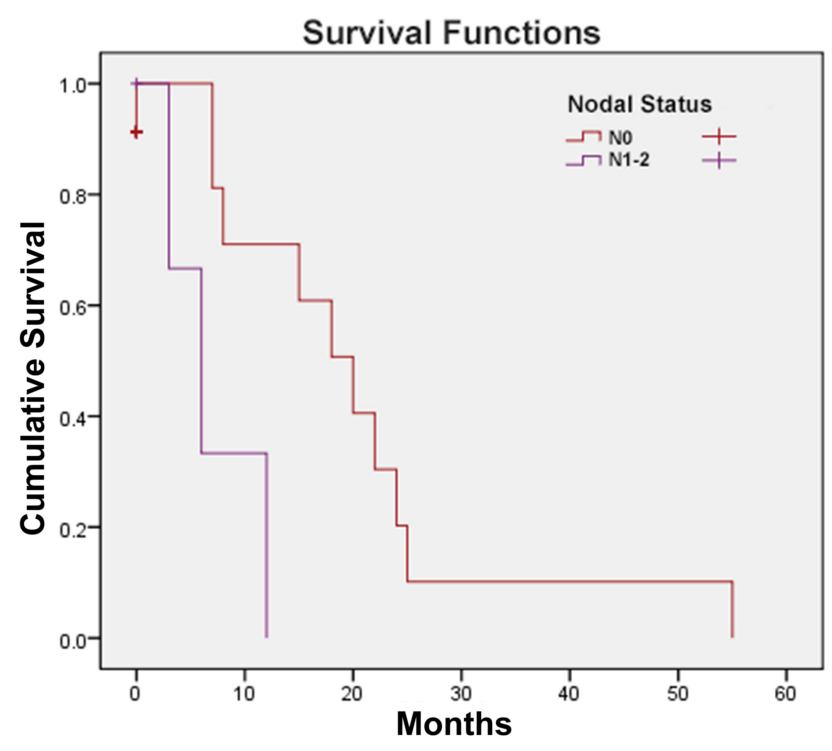

Figure 4 The survival probability according to lymph node status.

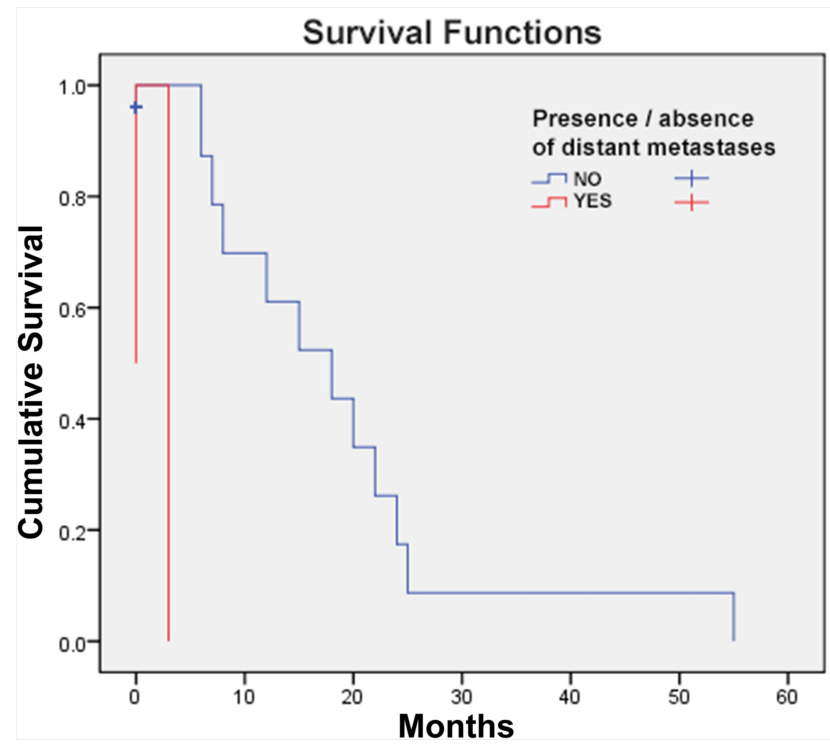

Figure $\mathbf{5}$ The survival probability according to the presence or absence of distant metastases.

a significant delay in cancer diagnosis. Factors involved in delaying the diagnosis of IBC vary from late referral of the patient to the doctor to general practitioners who must discern between a plethora of diagnoses. ${ }^{8}$

IBC includes signs of acute inflammation that can, at times, be misdiagnosed as infection, therefore any persistence of inflammatory signs lasting more than seven days under antibiotic and anti-inflammatory treatment should raise the suspicion of malignancy, and the patient must be referred to a surgeon in order to exclude malignancy. ${ }^{9}$ 


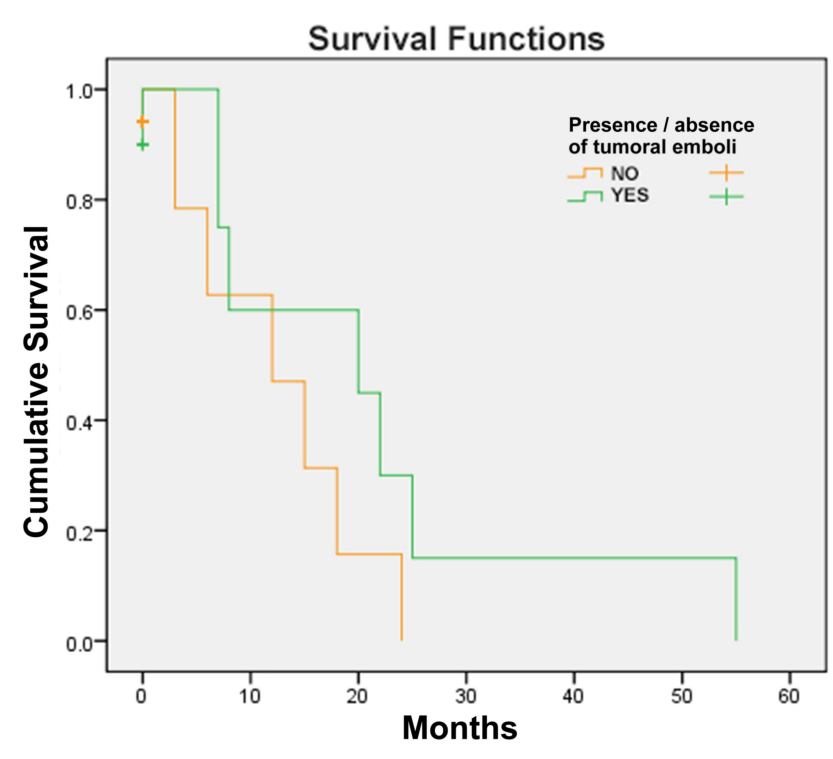

Figure 6 The survival probability according to the presence/absence of tumor emboli.

\section{Survival Functions}

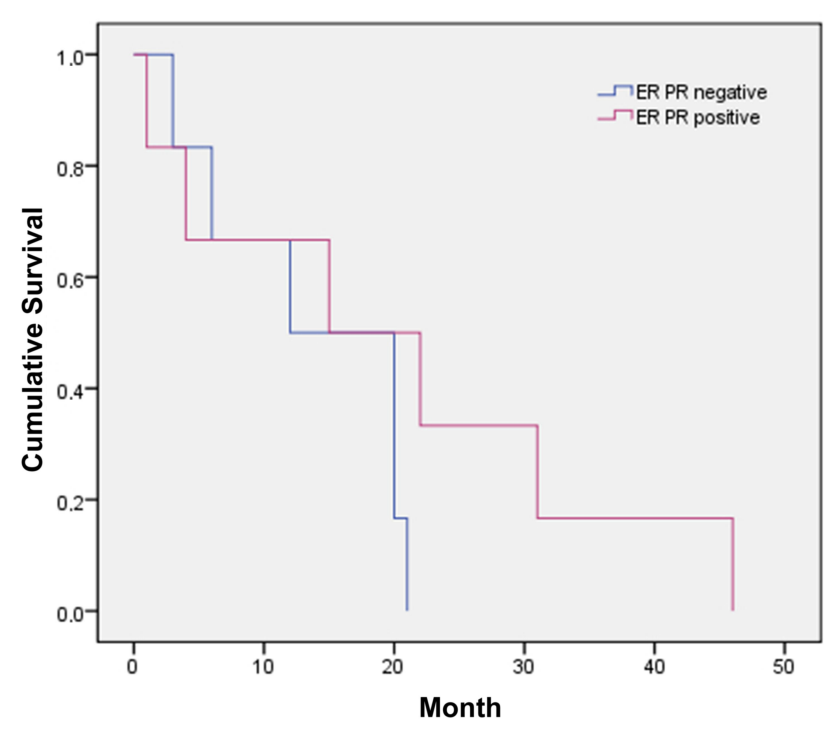

Figure 7 The survival probability according to the presence/absence of hormone receptors.

Clinical inflammation signs of IBC occur due to the presence of a tumor embolus and not as a consequence of an actual infection. ${ }^{10}$ In many cases, these clinical signs are misinterpreted as signs of infection not of malignancy by the patient and the general practitioner delaying the diagnosis of this form of breast neoplasia. $^{11}$

In the present study, the percentage of IBC cases was $2.26 \%$ of the total number of breast cancer cases.
Survival Functions

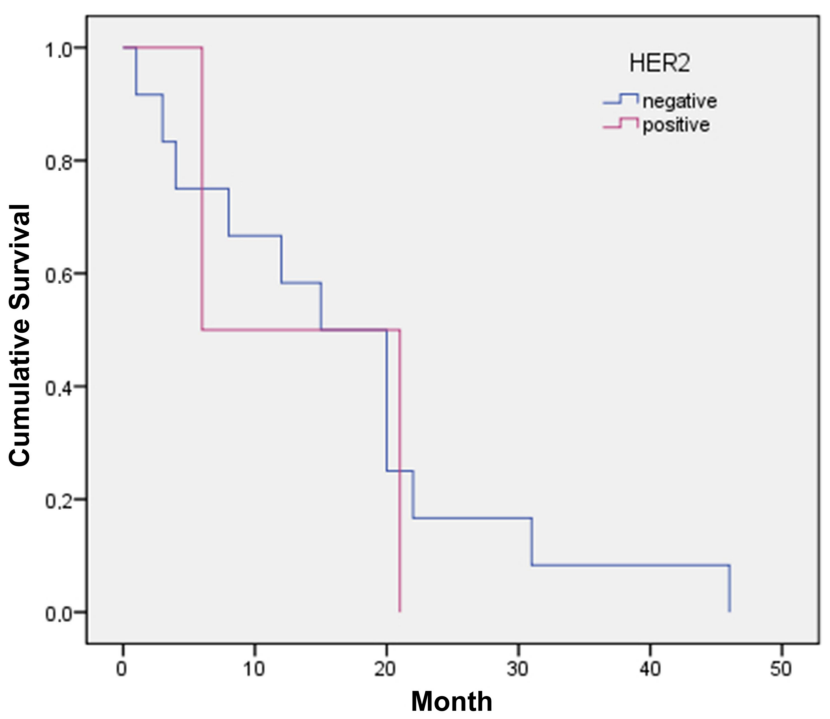

Figure 8 The survival probability according to HER2 overexpression.

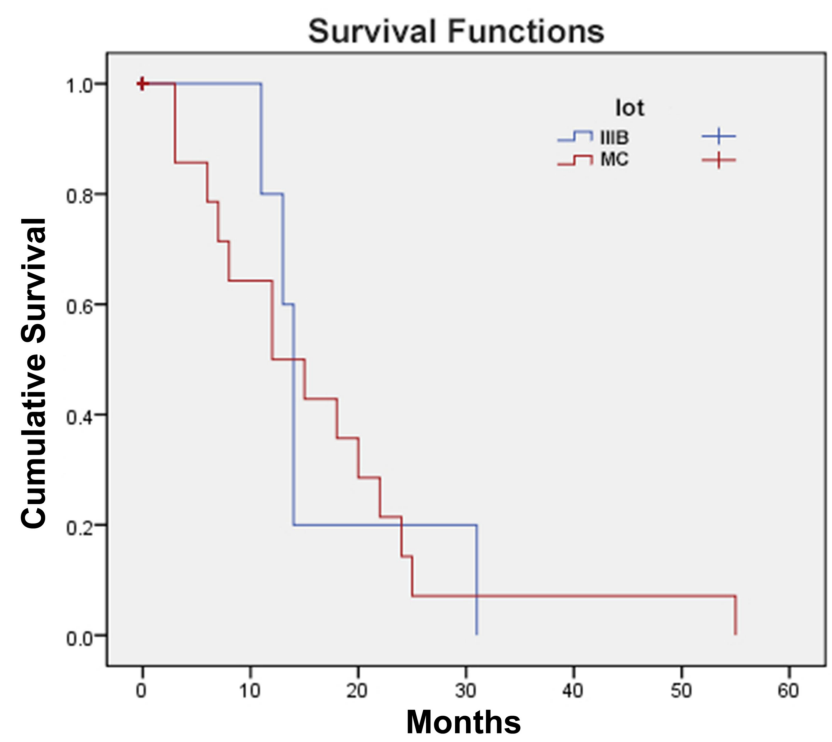

Figure 9 The survival probability of carcinomatous mastitis compared with stage IIIB noninflammatory breast cancer.

Similar percentages were published in an article from the USA, which reports a $2.5 \%$ incidence. ${ }^{12}$ In another study, the incidence of IBC varies from $0.6-2 \%$ of all breast cancer cases diagnosed each year. ${ }^{13}$ The incidence of IBC in Tunisia was $6 \%$ according to previous studies, while in Egypt, the percentage was $11 \%{ }^{14,15}$

The median age of patients with carcinomatous mastitis was 57 years, compared with the median age of the patients in stage IIIB NIBC, which was 66 years. Other 
studies note that IBC has a tendency of occurring in younger women (52 years), compared to other NIBC (57 years). ${ }^{16}$

Regarding the distribution of cases by histopathological type, $74.08 \%$ (20 patients) had infiltrating ductal carcinoma in the present study. These findings concur with another paper that studied 117 cases of carcinomatous mastitis and determined that infiltrating ductal carcinoma was predominant, followed by lobular carcinoma and other histological subtypes. ${ }^{15}$ Another US study that included 50 patients found that 47 patients $(94 \%)$ had infiltrating ductal carcinoma. $^{12}$

Tumor dimension in resection specimens was an important factor in clinical outcome. Patients with smaller than $5 \mathrm{~cm}$ tumors (ypT1-ypT2) had better survival probability than patients with bigger tumors (ypT3-ypT4). Other studies also conclude that tumor dimension is an important factor of the outcome in IBC. ${ }^{17}$

Consistent with the findings of numerous other authors, this study has determined that the number of months of survival increased significantly in cases that had an N0 stage compared to $\mathrm{N} 1$ cases and above. ${ }^{18,19}$

Only two $(7.40 \%)$ patients in this study had distant metastases, a lower percentage than that found in a study performed in the US, in which about $36 \%$ of patients with IBC had metastases at diagnosis. ${ }^{20}$ Dawood et al also found a higher presence of metastases (30\%). These differences in the number of patients with disseminated metastases could be explained by the smaller size of the study. Both women with metastatic disease died within a few months after surgery, but neither of these women had an uncontrolled local disease. Both this study and the one performed in the US over a period of four years proved that survivability dramatically decreases if disseminated metastases were present. ${ }^{6}$

There were no statistically significant differences between patients depending on the presence of tumor emboli (Mantel-Cox log rank test, $p=0.25, \alpha=0.05$ ). All cases were treated with neoadjuvant chemotherapy. We had no complete response (no T0), but we found 37.04\% of cases were tumor emboli free on resection of specimens, meaning partial response. The presence or absence of these emboli did not influence survival. Another study showed that the presence of lymph vessel tumor emboli in the advanced area of primary invasive tumors, and the presence of lymph vessel tumor emboli in the nontumor areas of primary invasive tumors were significantly associated with tumor recurrence but not tumor-related death. ${ }^{21}$
According to our study, hormone receptor-positive status was significant ( $p=0.043$ ), improving survival probability. Other studies showed similar conclusions, namely that the presence of hormone receptors improve the outcomes in IBC. $^{22}$

We have found no statistical difference in survival due to the overexpression of HER2 $(p=0.763)$. We must mention that the administration of trastuzumab as a neoadjuvant therapy combined with chemotherapy in patients with overexpression of HER2 was not a standard treatment before 2016 in our country, trastuzumab was only administered as an adjuvant therapy in HER2-positive cases. Other studies also found that survival was not linked to the HER2 status in the absence of trastuzumab as neoadjuvant treatment combined with chemotherapy. ${ }^{23}$

The 12-month survivability was similar between IBC and stage IIIB NIBC, the survival rate being $50 \%$ for patients with IBC, and $68 \%$ for NIBC. At 24 months, the differences become significant, as Anderson et al proved that survival rates were lower for the group with IBC (2237 patients, log rank test, $p<0.0001$ ) than for the group with NIBC. ${ }^{24}$ In another study performed between 1975 and 1977, and 1990 and 1992, survival rates at three years were $32 \%$ and $42 \%$, respectively for patients with IBC, and $80 \%$ and $85 \%$, respectively for patients with noninflammatory breast cancer. ${ }^{25}$

The limits of our study were the retrospective nature and the insufficient number of patients per subtype for analysis, resulting in limited statistical power, lack of data on the response rate to neoadjuvant chemotherapy and the possible progression of the disease under this treatment. However, all patients were included in the analysis (that is, even those with serious comorbidities and poor performance status). Our survival analysis was limited by the short follow-up period and also by the specific radiotherapy treatment.

\section{Conclusion}

Although nowadays there has been remarkable progress in early diagnosis, even before the clinical manifestations occur, IBC is often diagnosed at advanced stages, having a very reserved prognosis.

Negative outcome factors for the patients' survival when diagnosed with IBC are represented by tumor size $>5 \mathrm{~cm}$, the presence of metastases in axillary lymph nodes encountered in excision pieces, IBC hormone-receptor negative and the presence of distant metastases.

Neither the histopathological type nor the presence of tumor embolus influence the survival of patients with IBC 
according to the present study. Survival is not linked to the HER2 status in the absence of trastuzumab as treatment combined with neoadjuvant chemotherapy.

The prognosis of IBC patients is more reserved compared to NIBC.

Breast inflammation in the absence of lactation and especially during perimenopause age requires a proactive attitude including an appointment at the doctors' office or hospital for proper evaluation and diagnosis.

\section{Disclosure}

The authors report no conflicts of interest in this work.

\section{References}

1. A review of inflammatory breast cancer. https://www.uspharmacist. com/article/a-review-of-inflammatory-breast-cancer-43018. Accessed November 5, 2020. Accessed March 4, 2020.

2. Sabet S, Shrouk Khalaf ES, Hossam TM, Mohamed ES, Mona MM. Inflammatory breast cancer: high incidence of GCC haplotypes $(-1082 \mathrm{~A} / \mathrm{G},-819 \mathrm{~T} / \mathrm{C}$, and $-592 \mathrm{~A} / \mathrm{C})$ in the interleukin-10 gene promoter correlates with over-expression of interleukin-10 in patients' carcinoma tissues. Tumor Biol. 2017;39(7):10104283177 13393. doi: $10.1177 / 1010428317713393$.

3. Lim B, Woodward WA, Wang X, et al. Inflammatory breast cancer biology: the tumor microenvironment is key. Nat Rev Cancer. 2018;18:485-499. doi:10.1038/s41568-018-0010-y

4. Mercatali L, Spadazzi C, Miserocchi G, et al. The effect of everolimus in an in vitro model of triple negative breast cancer and osteoclasts". Int J Mol Sci. 2016;17(11):1827. doi:10.3390/ijms17111827

5. Brown L, Harmsen W, Blanchard M, et al. Once-daily radiation therapy for inflammatory breast cancer. Int J Radiat Oncol Biol Phys. 2014;89:997-1003. doi:10.1016/j.ijrobp.2014.01.054

6. Dawood S, Merajver SD, Viens P, et al. International expert panel on inflammatory breast cancer: consensus statement for standardized diagnosis and treatment. Ann Oncol. 2011;22(3):515-523.

7. Bonev V, Evangelista M, Chen JH, et al. Long-term follow-up of breast-conserving therapy in patients with inflammatory breast cancer treated with neoadjuvant chemotherapy. Am Surg. 2014;80 (10):940-943. doi:10.1177/000313481408001006

8. Robertson FM, Cristofanilli M. A global approach to inflammatory breast cancer. Future Oncol. 2011;7(1):25-30. doi:10.2217/fon.10.177

9. Rosa M. "Inflammatory" changes in breast: how to provide a better care to our patients. Arch Gynecol Obstet. 2010;281(5):901-905. doi:10.1007/s00404-009-1236-4

10. Nishimura R, Koyama H, Kasumi F, et al. A case control study on risk factors involved in inflammatory breast recurrence after breast-conserving surgery. Oncology. 1998;55(5):391-399. doi:10.11 $59 / 000011884$

11. van Uden DJP, Westenberg AHH, van Laarhoven HWM, Meijer JWR, de Wilt JHWH, Blanken-Peeters CFJM. [Mastitis as a symptom of malignancy]. Ned Tijdschr Geneeskd. 2014;158:A7591. Dutch.

12. Levine $\mathrm{PH}$, Zolfaghari L, Young $\mathrm{H}$, et al. What is inflammatory breast cancer? Revisiting the case definition. Cancers (Basel). 2010;2(1):143-152. doi:10.3390/cancers 2010143
13. Ionta MT, Atzori F, Massidda B. Inflammatory breast cancer in Italy: epidemiological and clinical aspects. Cancer. 2010;116:2736-2740. doi:10.1002/cncr.25164

14. Soliman AS, Banerjee M, Lo AC, et al. High proportion of inflammatory breast cancer in the population-based cancer registry of Gharbiah, Egypt. Breast J. 2009;15(4):432-434. doi:10.1111/j.15244741.2009.00755.x

15. Chaher N, Arias-Pulido H, Terki N, et al. Molecular and epidemiological characteristics of inflammatory breast cancer in Algerian patients. Breast Cancer Res Treat. 2012;131(2):437-444. doi:10.10 07/s10549-011-1422-5

16. Manfrin E, Remo A, Pancione M, et al. Comparison between invasive breast cancer with extensive peritumoral vascular invasion and inflammatory breast carcinoma: a clinicopathologic study of 161 cases. Am J Clin Pathol. 2014;142(3):299-306. doi:10.1309/ AJCPOXKX67KRAOVM

17. Biswas T, Jindal C, Timothy L, Fitzgerald JT. Pathologic Complete Response (pCR) and Survival of Women with Inflammatory Breast Cancer (IBC): an analysis based on biologic subtypes and demographic characteristics. Int J Environ Res Public Health. 2019;16 (1):124. doi:10.3390/ijerph16010124

18. Gajdos C, Tartter PI, Estabrook A, Gistrak MA, Jaffer S, Bleiweiss IJ. Relationship of clinical and pathologic response to neoadjuvant chemotherapy and outcome of locally advanced breast cancer. J Surg Oncol. 2002;80(1):4-11. doi:10.1002/jso.10090

19. Machiavelli MR, Romero AO, Pérez JE, et al. Prognostic significance of pathological response of primary tumor and metastatic axillary lymph nodes after neoadjuvant chemotherapy for locally advanced breast carcinoma. Cancer J Sci Am. 1998;4(2):125-131.

20. Kleer CG, van Golen KL, Merajver SD. Molecular biology of breast cancer metastasis. Inflammatory breast cancer: clinical syndrome and molecular determinants. Breast Cancer Res. 2000;2(6):423-429. doi:10.1186/bcr89

21. Tamura N, Hasebe T, Okada N. Tumor histology in lymph vessels and lymph nodes for the accurate prediction of outcome among breast cancer patients treated with neoadjuvant chemotherapy. Cancer Sci. 2009;100(10):1823-1833. doi:10.1111/j.1349-7006.2009.01264

22. Li J, Yue X, Wu Q, et al. Outcomes of patients with inflammatory breast cancer by hormone receptor- and HER2-defined molecular subtypes: a population-based study from the SEER program. Oncotarget. 2017;8(30):49370-49379.

23. Gianni L, Eiermann W, Semiglazov V, et al. Neoadjuvant chemotherapy with trastuzumab followed by adjuvant trastuzumab versus neoadjuvant chemotherapy alone, in patients with HER2-positive locally advanced breast cancer (the NOAH trial): a randomized controlled superiority trial with a parallel HER2-negative cohort. Lancet. 2010;375(9712):377-384. doi:10.1016/S0140-6736(09)61964-4

24. Anderson WF, Chu KC, Chang S. Inflammatory breast carcinoma and noninflammatory locally advanced breast carcinoma: distinct clinicopathologic entities? J Clin Oncol. 2003;21(12):2254-2259. doi:10.1200/JCO.2003.07.082

25. Chang S, Parker SL, Pham T, Buzdar AU, Hursting SD. Inflammatory breast carcinoma incidence and survival: the surveillance, epidemiology, and end results program of the National Cancer Institute, 1975-1992. Cancer. 1998;82(12):2366-2372. 


\section{Publish your work in this journal}

Cancer Management and Research is an international, peer-reviewed open access journal focusing on cancer research and the optimal use of preventative and integrated treatment interventions to achieve improvec outcomes, enhanced survival and quality of life for the cancer patient.
The manuscript management system is completely online and includes a very quick and fair peer-review system, which is all easy to use. Visit http://www.dovepress.com/testimonials.php to read real quotes from published authors. 